\title{
Modeling of Fire Spread Including Different Heat Transfer Mechanisms Using Cellular Automata
}

\author{
Jarosław Wąs $\left.{ }^{(}\right)(10)$, Artur Karp, Szymon tukasik, and Dariusz Pałka@ \\ Faculty of Electrical Engineering, Automatics, IT and Biomedical Engineering, \\ AGH University of Science and Technology, \\ Mickiewicza 30, 30-059 Krakow, Poland \\ jaroslaw.was@agh.edu.pl
}

\begin{abstract}
The article presents a new method of modeling the spread of fire using Cellular Automata based on 3D mesh. The models based on different heat transport mechanisms were used in the study, including conductivity, convection and thermal radiation. General mechanisms of fire spread are exemplified by a fire inside a building. The tests of the created simulator for fire in rooms were conducted, and the reference point was the known simulator based on CFD, namely FDS Fire Dynamics Simulator.
\end{abstract}

Keywords: Fire spread $\cdot$ Cellular Automata $\cdot$ Heat transfer mechanisms

\section{Introduction}

Fire is a complex phenomenon which includes thermal degradation or pyrolysis of solid fuel into gas (of a volatile character) and combustion of the volatile fuel. It should be noted here that the generated heat influences the solid. Heat transport is carried out through thermal conductivity, convection (natural and forced) and thermal radiation. Modern computational sciences provide various methods and IT tools that allow for modeling this complex process [8] in a simplified way.

The most common models are high-fidelity CFD models, also known as Field models, like e.g. Fire Dynamics Simulator (FDS). based on the physical principles of conservation of: mass, energy, and momentum. In the field model, the motion of heat and smoke is calculated in particular cells in consecutive time intervals. In such models we assume that in each cell the properties like temperature, density, flow, etc. are constant. Another popular approach are Zone models, in which the whole space (for instance, a room) is divided into areas with different properties (like a 'combustion zone' or a 'convection zone'), and for each of them a set of algebraic equations is solved in order to calculate relevant flow properties. The most recent approach assumes looking for fire models that are realistic and computationally efficient.

The paradigm of Cellular Automata (CA) can be considered an effective and efficient solution. In the literature, $\mathrm{CA}$ are used to model simplified fire spreading

(C) Springer Nature Switzerland AG 2020

V. V. Krzhizhanovskaya et al. (Eds.): ICCS 2020, LNCS 12137, pp. 445-458, 2020.

https://doi.org/10.1007/978-3-030-50371-0_33 
schemes [1], often in relation to evacuation [13] or specific scenarios like forest fires $[6,14]$ etc.

We propose a new model of fire spread based on Cellular Automata in which different mechanisms of heat transfer are taken into account, namely: conductivity, convection and thermal radiation. We propose the application of the cuboid structure of space using three-dimensional CA.

We developed a theoretical model, implemented it, and performed preliminary validation based on widely used CFD model FDS, created by NIST (National Institute of Standards and Technology), which is often applied as a reference model $[4,10]$

\section{Heat Transfer Mechanisms}

\subsection{Thermal Conduction}

The amount of energy which is transferred in thermal conduction can be computed using the law of heat conduction (Fourier's law). The differential form of this law is as follows:

$$
q=-k \nabla T
$$

where: $q$ - is local heat flux density (SI unit $\left[\frac{W}{m^{2}}\right]$ ), $k$ - is material conductivity (SI unit $\left[\frac{W}{m K}\right]$ ), $\nabla T$ - is the temperature gradient (SI unit $\left[\frac{K}{m}\right]$ ).

\subsection{Thermal Radiation}

The heat flux that can be emitted by radiation coming from the black body surface is given by Stefan-Boltzmann law:

$$
q=\sigma T^{4}
$$

where: $q-$ is radiative heat flux (SI unit $\left[\frac{W}{m^{2}}\right]$ ), $\sigma-$ is the Stefan-Boltzmann constant $\left(\approx 5.670 \cdot 10^{-8}\left[\frac{W}{m^{2} K^{4}}\right]\right), T-$ is the temperature of the body surface (SI unit $[K])$

The flux emitted by the real surface is lower than the flux emitted by the black body surface:

$$
q=\epsilon \sigma T^{4}
$$

where: $\epsilon$ - a factor called emissivity, which is a property characterising a surface $(\epsilon<1)$

\subsection{Convection}

Convective heat transfer is transfer of heat from one place to another caused by the movement of fluid. The rate equation for this form of heat transfer is described by Newton's law of cooling:

$$
q=h\left(T-T_{f}\right)
$$

where: $q$ - is convective heat flux (SI unit $\left[\frac{W}{m^{2}}\right]$ ), $h$ - is convection heat transfer coefficient (SI unit $\left.\left[\frac{W}{m^{2} K}\right]\right),\left(T_{w}-T_{f}\right)$ - is the temperature difference between the surface of an object and fluid (SI unit $[K]$ ). 


\section{CAFire}

The assumptions of the model used in the implementation of the CAFire programme which simulates fire spread are presented below.

The whole space under consideration can be divided into three-dimensional elements (e.g. cubes) small enough to be treated as homogeneous - i.e. all physical and chemical properties (e.g. temperature, specific heat capacity) in a given element are the same at a given time.

\subsection{Neighborhood}

Three basic types of neighborhoods exist in a three-dimensional array of a cubic cell:

- 6 cells sharing faces with the reference cell

- 18 cells sharing edges with the reference cell

- 26 cells sharing vertices with the reference cell

Because the simulation results obtained with the use of all the above types of neighborhoods are comparable and the first variant has the lowest calculation costs, it is used in the model (Fig. 1).

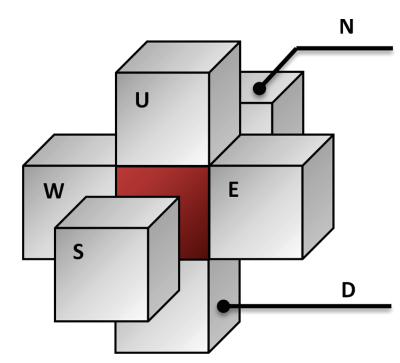

Fig. 1. Neighborhood. Red - the reference cell, U - the cell above, D - the cell below, $\mathrm{W}$ - the cell on the left, $\mathrm{E}$ - the cell on the right, $\mathrm{S}$ - the cell in front, $\mathrm{N}$ - the cell behind. (Color figure online)

\subsection{Simulation of Heat Transfer Using the CA Model}

Thermal Conduction. At a given temperature gradient, the heat transfer rate is proportional to the surface through which the heat flows. Generally, the amount of heat $d Q$ that will flow $L$ over $d t$ with the temperature difference $d T$ across the transverse surface $S$ is as follows:

$$
d Q=\lambda \cdot \frac{S}{L} \cdot d t \cdot d T
$$


where: $Q$ - heat, $\lambda$-thermal conductivity coefficient, $S$ - transverse surface of the cell, $L$ - distance between cells, $d t$ - time, $d T$ - temperature difference.

In the proposed model, the cells have the same surface area. Consider the following one-dimensional situation shown in Fig. 2.

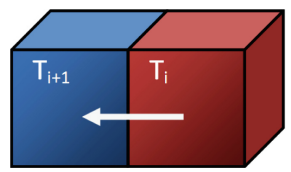

$$
\begin{aligned}
& \square \text { - warmer cell } \\
& \square \text { - cooler cell }
\end{aligned}
$$

Fig. 2. Heat flow between two neighbouring cells due to thermal conductivity in the model.

In this type of analyses, it is assumed that the heat exchange occurs between the cells, and so the $\lambda$ factor coefficient indicates how the heat passes from one cell to another.

With two cells only one factor is determined. However, if a whole cell chain is considered, it may happen that for a given cell $n$ the conduction coefficient from/to cell $(n-1)$ is different than from/to $(n+1)$.

In the discrete one-dimensional case, the formula for temperature $T$ of the cell with index $n$ and of a unit size can be written as:

$$
T_{n}(t+\Delta t)=T_{n}(t)+\left(\lambda_{n}^{\prime} \cdot\left(T_{n}(t)-T_{n-1}(t)\right)-\lambda_{n+1}^{\prime} \cdot\left(T_{n+1}(t)-T_{n}(t)\right)\right) \Delta t
$$

where: $\lambda^{\prime}=\frac{\lambda}{m \cdot c}, T_{n}(t)$ - Cell $n$ temperature at a given time $t, \lambda$ - thermal conductivity coefficient, $c$ - specific heat capacity, $m$ - cell mass.

\subsection{Convection}

The phenomenon of convection is characteristic of liquids and gases. The model includes natural convection.

$$
\begin{aligned}
& T_{u}(t+\Delta t)=T_{u}(t)+\alpha^{\prime} \cdot\left(T_{u}(t)-T_{d}(t)\right) \cdot \Delta t \\
& T_{d}(t+\Delta t)=T_{d}(t)-\alpha^{\prime} \cdot\left(T_{u}(t)-T_{d}(t)\right) \cdot \Delta t
\end{aligned}
$$

where: $\alpha^{\prime}=\frac{\alpha}{m \cdot c}, T_{u}(t)$ - the temperature of the upper cell at a given time $t$, $T_{d}(t)$ - the temperature of the lower cell at a given time $t, \alpha$-convection heat transfer coefficient, $m$ - cell mass, $c$ - specific heat capacity (Fig. 3). 


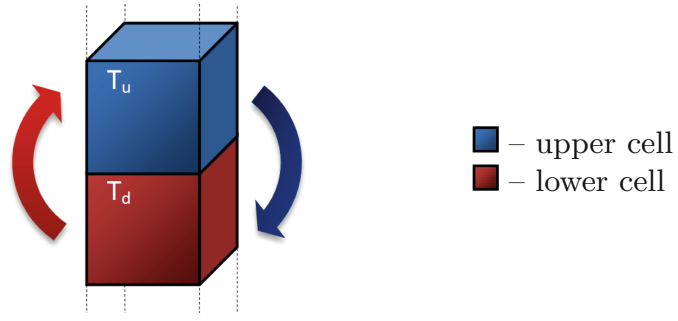

Fig. 3. Convection in the model. Heat exchange from the cell below to the cell above. $T_{d}$ - the temperature of the lower cell, $T_{u}$ - the temperature of the upper cell

\subsection{Thermal Radiation}

Thermal radiation is a process in which energy is emitted by a heated surface in all directions in the form of electromagnetic waves (electromagnetic radiation).

In the fire safety literature, thermal radiation has been identified as important and even dominant in terms of heat transfer in medium to large fires. Its mechanisms are analysed at various levels of accuracy ranging from simplified analyses to sophisticated methods of calculating radiation [9].

Stefan-Boltzmann law refers to radiation in all directions, whereas in the model only some of the energy radiated by one surface will reach another. The heat exchange from a warmer (1) to a cooler (2) surface depends on the following factors [5]:

- temperatures $T_{1}$ and $T_{2}$

- surface areas $A_{1}$ and $A_{2}$

- spatial configurations between surfaces

- surface radiation characteristics

- additional surfaces in the environment

- medium between surfaces (if it is air, it can be assumed as non-invasive)

To calculate the amount of energy that will reach from one surface to another, we need to calculate the integral, taking into account the density of the radiation at an angle under which fragments of the radiating surface are visible for the other surface. Then, an additional factor view factor appears in StefanBoltzmann law.

$$
Q_{n e t}=F_{1-2} A_{1} \sigma\left(T_{1}^{4}-T_{2}^{4}\right)
$$

where: $Q_{n e t}$ - total radiation energy, $F_{1-2}$ - view factor, $A_{1}$ - surface area, $\sigma-$ Stefan - Boltzmann constant, $T_{1}, T_{2}$ - temperature in Kelvins.

The building is divided into radiation areas (for example, walls or tables) with a direction from which they can receive heat (Figs. 4 and 5). 


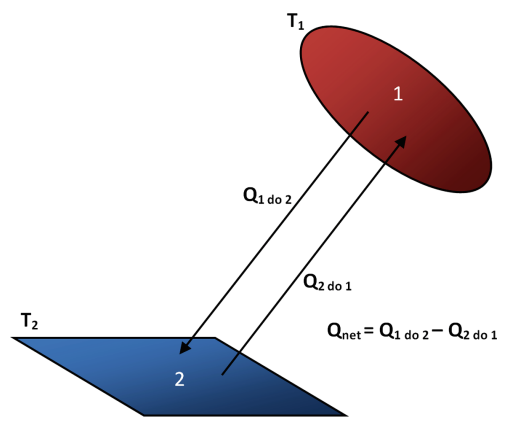

Fig. 4. General radiation pattern. $Q_{n e t}$ - total energy exchange, $Q_{x}$ do $y$ - energy transferred from the surface $x$ to $y, T_{x}$ - temperature of surface $x$.

\section{View Factors for Several Three-Dimensional Configurations [5]}

- Parallel surfaces

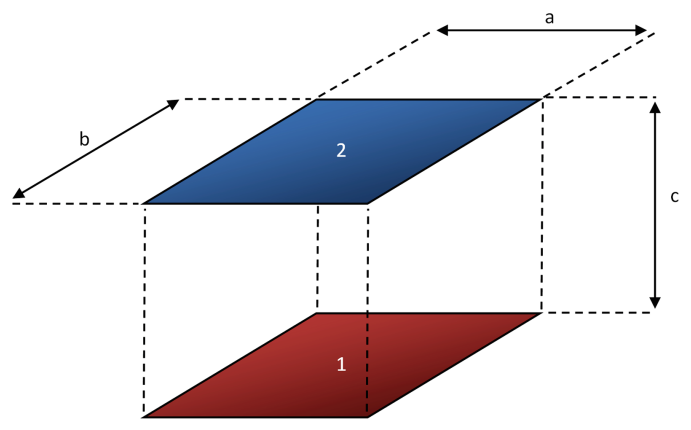

Fig. 5. Diagram of radiation for parallel surfaces in the model. $a / b$ - length/width of surfaces, $c$ - distance between surfaces

let:

$$
X=\frac{a}{c} \text { i } Y=\frac{b}{c}
$$

then:

$$
\begin{aligned}
F_{1-2}= & \frac{2}{\pi X Y}\left(\ln \left(\frac{\left(1+X^{2}\right)\left(1+Y^{2}\right)}{1+X^{2}+Y^{2}}\right)^{\frac{1}{2}}-X \tan ^{-1} X-Y \tan ^{-1} Y\right. \\
& \left.+X \sqrt{1+Y^{2}} \tan ^{-1} \frac{X}{\sqrt{1+Y^{2}}}+Y \sqrt{1+X^{2}} \tan ^{-1} \frac{Y}{\sqrt{1+X^{2}}}\right)
\end{aligned}
$$

where: $F_{1-2}$ - view factor, $a$ - length of surface, $b$ - width of surface, $c$ distance between surfaces (Fig. 6). 


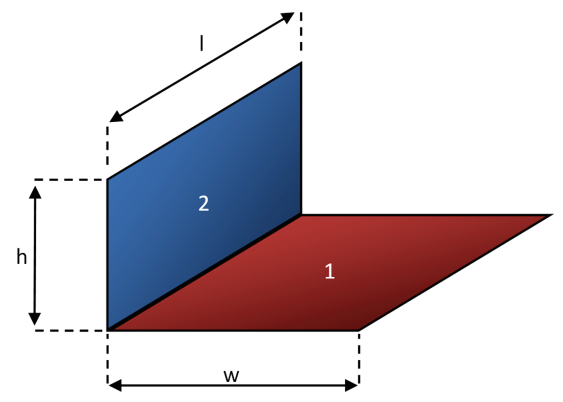

Fig. 6. Scheme of radiation for perpendicular surfaces in the model. $h$ - width of the first surface, $w$ - width of the second surface, $l$ length of the surface.

- Perpendicular surfaces let:

$$
H=\frac{h}{l} \text { i } W=\frac{w}{l}
$$

then:

$$
\begin{array}{r}
F_{1-2}=\frac{1}{\pi W}\left(W \tan ^{-1} \frac{1}{W}-\sqrt{H^{2}+W^{2}} \tan ^{-1}\left(H^{2}+W^{2}\right)^{-1 / 2}\right. \\
+H \tan ^{-1} \frac{1}{H}+\frac{1}{4} \ln \left(\left(\frac{\left(1+W^{2}\right)\left(1+H^{2}\right)}{1+W^{2}+H^{2}}\right)\right. \\
\left.\left.\left(\frac{W^{2}\left(1+W^{2}+H^{2}\right.}{\left(1+W^{2}\right)\left(W^{2}+H^{2}\right)}\right)^{W^{2}}\left(\frac{H^{2}\left(1+H^{2}+W^{2}\right)}{\left(1+H^{2}\right)\left(H^{2}+W^{2}\right)}\right)^{H^{2}}\right)\right)
\end{array}
$$

where: $F_{1-2}$ - view factor, $h$ - width of the first surface, $w$ - width of the second surface, $l$ - length of the surface

For each wall of a fire cell parallel radiation surfaces are considered where no fire cells or solid body cells are located between them in a straight line.

$T_{\text {surface }}$ was calculated as the average temperature of the cells contained in this surface. Of course, the following condition must be met: $T_{\text {cell }}>T_{\text {surface }}$.

As an obstacle, we took into account a cell which was a solid body or a fire cell. Knowing surface dimensions $a$ i $b$ and the distance from the cell $c$ coefficient, the view factor was calculated on the basis of Table 1.

The last step was to calculate the transferred energy $Q_{n e t}$ according to Eq. 8 . Energy is transferred equally to all cells belonging to the radiation surface.

In case of radiation surface $\Rightarrow$ surface, the main radiation surfaces were determined at the beginning of the simulation; they can be generally treated as walls (in the model for each room, 6 such surfaces were designated for the floor, 
Table 1. View factor values applied in CAFire simulation

\begin{tabular}{l|l|l|l|l|l|l|l}
\hline \multirow{2}{*}{$\frac{a}{c}$} & \multicolumn{7}{l}{$\frac{b}{c}$} \\
\cline { 2 - 9 } & $<0.1$ & 0,2 & 0,3 & 0,4 & 0,6 & 1 & $2<$ \\
\hline$<0,1$ & 0,003 & 0,006 & 0,008 & 0,012 & 0,016 & 0,021 & 0,028 \\
\hline 0,2 & 0,005 & 0,012 & 0,018 & 0,022 & 0,031 & 0,040 & 0,051 \\
\hline 0,3 & 0,009 & 0,018 & 0,026 & 0,033 & 0,045 & 0,059 & 0,074 \\
\hline 0,4 & 0,012 & 0,022 & 0,033 & 0,042 & 0,059 & 0,075 & 0,091 \\
\hline 0,6 & 0,015 & 0,030 & 0,045 & 0,059 & 0,078 & 0,100 & 0,145 \\
\hline 1 & 0,020 & 0,039 & 0,057 & 0,075 & 0,105 & 0,150 & 0,170 \\
\hline $2<$ & 0,025 & 0,048 & 0,071 & 0,092 & 0,125 & 0,175 & 0,225 \\
\hline
\end{tabular}

ceiling and each wall). For each surface other surfaces in its "field of visions" and its view factors were determined on the basis of Eq.10 or 12. The same procedure was repeated in case of cell $\Rightarrow$ surface.

\subsection{Simulation of Fire Propagation Using the CA Model}

The proposed model takes into account the properties of relevant materials, because each of them has a different autoignition and ignition temperature.

Ignition is possible if a cell receives temperature proper to a given kind of material (e.g. wood $250^{\circ} \mathrm{C}$, carpet $100{ }^{\circ} \mathrm{C}$, fabrics $170^{\circ} \mathrm{C}$ ) and one of the neighboring cells remains on fire long enough (Fig. 7 ).

The only condition for autoignition is to reach a sufficiently high temperature (e.g. wood $2000^{\circ} \mathrm{C}$, carpet $500^{\circ} \mathrm{C}$, fabrics $650^{\circ} \mathrm{C}$ ).

The linear fire spread rate was calculated for each material. By transforming the formula to take into account the speed, we calculated how long the cell must burn (to be able to ignite its neighbor) (Fig. 8).

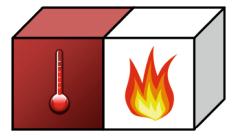

$$
\begin{aligned}
& \text { If }\left(T>T_{\text {ignition }}\right. \text { and } \\
& \text { burning time }>\text { ignition time) } \\
& \text { set in the cell "fire" (red) state. }
\end{aligned}
$$

Fig. 7. Interpretation of cell ignition. White cell - a cell with temperature that ignites, red cell - an adjacent cell burning for an appropriate period of time. (Color figure online)

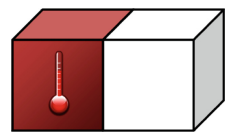

If $\left(T>T_{\text {autoignition }}\right)$ set in the cell "fire" (red) state.

Fig. 8. Interpretation of cell autoignition. Red cell - a cell that is autoignited (without burning neighbors). (Color figure online) 


\subsection{Simulation of Smoke Propagation Using the CA Model}

In the presented model smoke is generated in the cell located directly above the flame, which is shown in Fig. 9. The amount of smoke emitted depends on the type of material that is burning. For example, less smoke will be released from a wooden cell than from a textile cell.

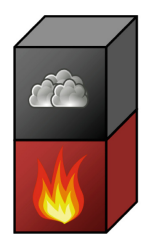

$\square$ - cell with smoke
$\square$ - cell with fire

Fig. 9. Smoke release mechanism (I). Smoke is generated in the cells located directly above the cells with fire.

The principle of mass conservation and the convection mechanism were retained in smoke modeling.

It was assumed that every cell tends to get rid of all its smoke. To ensure that the cell is not filled above its maximum capacity, the amount of smoke that it can take from one neighbor was limited to $\frac{1}{6}$ of the remaining free space [12].

In order to ensure that the cell does not transmit more smoke than it has, the amount of smoke that a given cell can transmit was limited to $\frac{1}{6}$ of the amount of smoke.

$$
D_{0 \rightarrow n}=d_{n} \cdot \min \left(\frac{1}{6} D_{0}, \frac{1}{6}\left(100 \%-D_{n}\right)\right)
$$

The amount of smoke in the cell will be reduced by the amount the cell will pass on to its neighbors and increased by the amount it will receive from its neighbors (Fig. 10):

$$
D_{0}^{i+1}=D_{0}^{i}-\sum_{n} D_{0 \rightarrow n}^{i}+\sum_{n} D_{n \rightarrow 0}^{i}
$$

where:

$D_{0 \rightarrow n}$ - the amount of smoke passed to a neighbouring cell

$D_{n \rightarrow 0}$ - the amount of smoke received from a neighbouring cell

$d_{n}-$ quantitative factor, according to Table 2

$n$ - neighbor index 
Table 2. Smoke propagation factor $d_{n}(1)$

\begin{tabular}{l|r}
\hline$n$ & $d_{n}$ \\
\hline $\mathrm{U}$ & $100 \%$ \\
\hline $\mathrm{N}, \mathrm{S}, \mathrm{E}, \mathrm{W}$ & $50 \%$ \\
\hline $\mathrm{D}$ & $25 \%$ \\
\hline
\end{tabular}

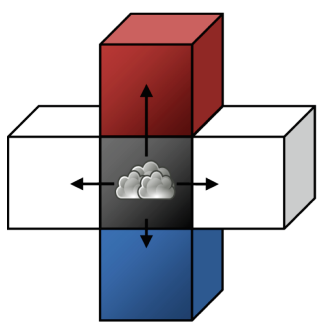

$$
\begin{aligned}
& \square \text { - cell above } \\
& \square \text { - cell with smoke } \\
& \square \text { - adjacent cell (N, E, S, W) } \\
& \square \text { - cell below }
\end{aligned}
$$

Fig. 10. Smoke generation mechanism. Propagation of smoke from a given cell ('the cell with smoke') to neighboring cells.

When the cell above $C_{U}$ is not air, or the amount of smoke in it $D_{U}$ is close to $100 \%$ (that is, it cannot accept more smoke), the $d_{n}$ factor will be modified (see Table 3).

This modification adequately demonstrates pushing smoke out of the ceiling zones.

\section{Calibration and Validation}

While calibrating fire scenarios, we took into account bibliographical data: standard PN-EN ISO 6946 and [3], as well as some data from reference fires [2]. The simulation was based on materials that are usually found in a typical household - Fig. 11 (Table 4).

Table 3. Smoke propagation factor $d_{n}(2)$

\begin{tabular}{l|l}
\hline$n$ & $d_{n}$ \\
\hline $\mathrm{U}$ & - \\
\hline $\mathrm{N}, \mathrm{S}, \mathrm{E}, \mathrm{W}$ & $50 \%$ \\
\hline $\mathrm{D}$ & $100 \%$ \\
\hline
\end{tabular}


Table 4. Material properties table - simulation.

\begin{tabular}{l|l|l|l|l|l|l}
\hline & Air & Wall ext. & Glass & Wood & Carpet & Fabrics \\
\hline Solid state $\mathrm{t}=21^{\circ} \mathrm{C}$ & No & Yes & Yes & Yes & Yes & Yes \\
\hline Combustibility & - & No & No & Yes & Yes & Yes \\
\hline Density $\left(\mathrm{kg} / \mathrm{m}^{3}\right)$ & $1.29^{* *}$ & 2200 & 2500 & 400 & 300 & 400 \\
\hline Specific heat $(\mathrm{J} /(\mathrm{kg} \cdot \mathrm{K}))$ & 1005 & 900 & 8400 & 2390 & 5200 & 2390 \\
\hline Conduction factor $(\lambda)$ & 0.026 & $0.250(0.550)$ & 0.500 & 0.300 & 0.174 & 0.300 \\
\hline Flash point $\left({ }^{\circ} \mathrm{C}\right)$ & - & - & $300^{*}$ & 250 & 120 & 350 \\
\hline Temp. of autoignition $\left({ }^{\circ} \mathrm{C}\right)$ & - & - & - & 2000 & 750 & 1400 \\
\hline Linear speed $(\mathrm{m} / \mathrm{s})$ & - & - & - & 3.0 & 0.8 & 3.0 \\
\hline Smoke emission & - & - & - & 0.3 & 0.8 & 1.0 \\
\hline Generated energy $(\mathrm{J} / \mathrm{s})$ & - & - & - & 1500 & 500 & 1500 \\
\hline
\end{tabular}

* - Since glass does not have a flashpoint, this value indicates the temperature at which the glass breaks.

** - At 1013.25 hPa and 0 degrees Celsius

\subsection{Validation of the Model}

The proposed model was validated in two ways: on the basis of movies recorded for fires taking place under controlled conditions, and on the basis of the results obtained with the Fire Dynamics Simulator tool. In case of movies, the spread

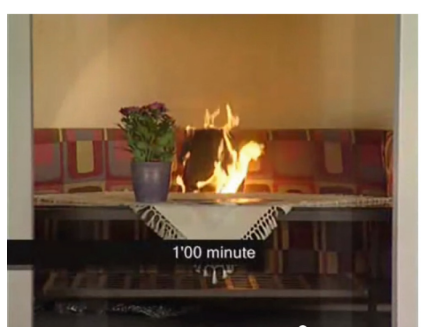

Movie 1

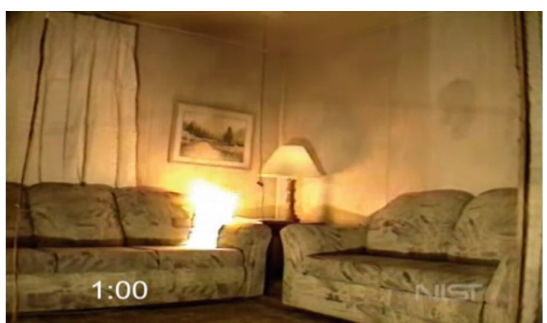

Movie 2

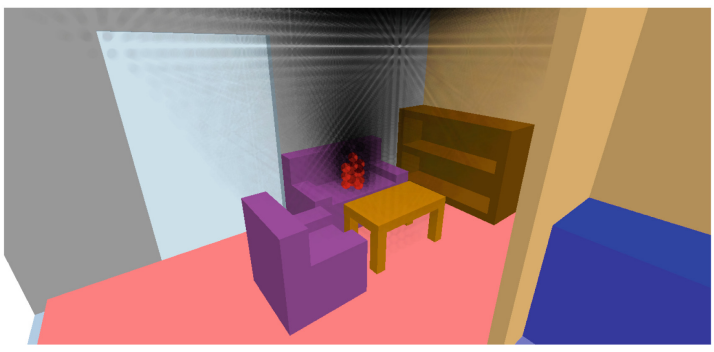

Model CAFire

Fig. 11. Simulation based on the data obtained from the reference fire ('Movie 1' and 'Movie 2') - time 1:00 after ignition. 
of fire and smoke was visually compared, while in case of FDS, the distribution of the temperatures in selected fire areas was additionally validated.

Fire Dynamics Simulator $[3,4,7,11]$ is an application that, according to many experts in fire engineering, best reflects the course of fire in buildings. In order to compare our CA-based model with the same model in FDS, we compared two identical scenarios of a building fire. The size of the building: $7.5 \mathrm{~m} \times 2.9 \mathrm{~m} \times$ $7.5 \mathrm{~m}$, including a living-room, a bathroom, a wardrobe, a study, a kitchen and a hall. Ignition temperature was $150{ }^{\circ} \mathrm{C}$.

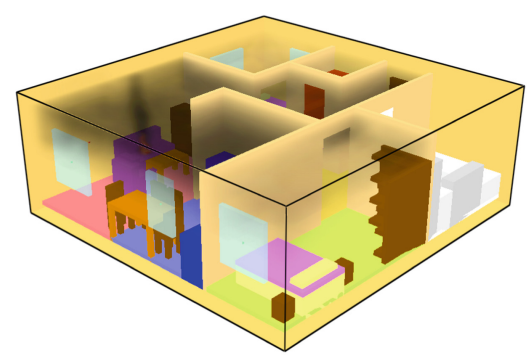

FDS time 01:00 min

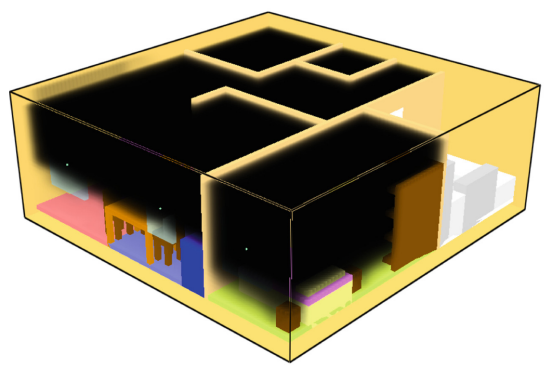

FDS time 05:00 min

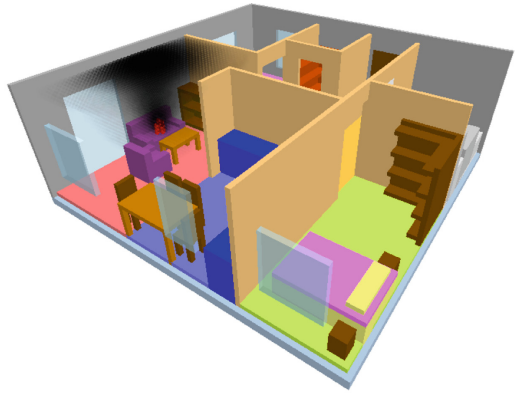

Model CAFire time 01:00 min

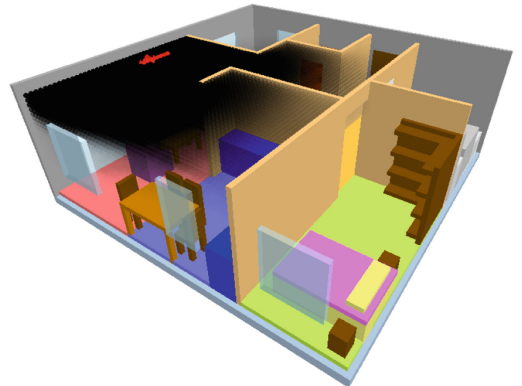

Model CAFire time 05:00 min

Fig. 12. Validation of fire and smoke spreading on the basis of CFD Fire Dynamics Simulator. The same configuration (scene) evaluated using FDS and Model CAFire.

It should be noted that other data sets were used for the calibration and validation procedures. Both, fire development scenario and basic parameters are similar in our model and the reference FDS model. Sample comparison results are presented in Fig. 12 and 13. 


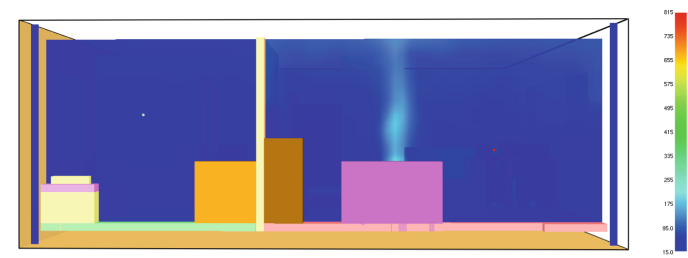

FDS

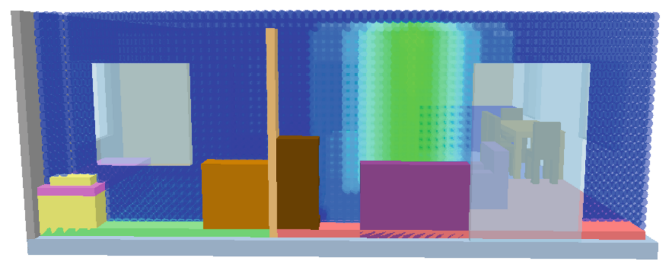

Model CAFire

Fig. 13. Validation on the basis of CFD Fire Dynamics Simulator - temperature distribution.

\section{Conclusions}

The main purpose of this study was to create an effective and efficient method for simulating the spread of fire. The model and simulations were based on nonhomogeneous Cellular Automata to present the distribution of fire and smoke in a residential building, taking into account the temperature fields in the rooms. The current model of fire called CAFire includes three main phenomena accompanying the spread of heat during a fire, i.e. conductivity, convection and thermal radiation. The simulations showed relatively reliable convergence of the results compared to the reputable FDS tool.

In the presented model, only in a very simplified form, the fluid flow phenomena (air flow, smoke flow) are simulated. The interactions between the air streamlines (due to Bernoulli's principle) and the influence of pressure caused by the difference of temperatures on the formation of laminar and turbulent flows etc. are not taken into account.

The idea presented in the paper requires further development and tests and additional emphasis should be placed on the chemical aspects of fire and exhaustive validation of the final results.

However, it should be emphasized that we consider the proposed direction of work very promising. Creating simulations in the FDS program requires time, even up to several dozen minutes, while calculations obtained using the proposed Cellular Automata method take seconds to be completed. 


\section{References}

1. Curiac, D.I., Banias, O., Volosencu, C., Dan, P.: Cellular automata based simulation for smoke and fire spreading in large buildings. In: International Conference on Development, Energy, Environment, Economics - Proceedings, November 2010

2. Fires, R.: Living room fires. http://www.youtube.com/watch?v=TB42Ib3A4mg

3. Hurley, M.J., et al. (eds.): SFPE Handbook of Fire Protection Engineering. Springer, New York (2016). https://doi.org/10.1007/978-1-4939-2565-0

4. Kang, D.I., Kim, K., Jang, S.C., Yoo, S.Y.: Risk assessment of main control board fire using fire dynamics simulator. Nucl. Eng. Des. 289, 195-207 (2015). https:// doi.org/10.1016/j.nucengdes.2015.04.030

5. Lienhard, J.: A heat transfer textbook. J. Heat Transf. 108 (2013)

6. Liu, Y., Liu, H., Zhou, Y., Sun, C.: Spread vector induced cellular automata model for real-time crown fire behavior simulation. Environ. Modell. Softw. 108, 14-39 (2018). https://doi.org/10.1016/j.envsoft.2018.07.005

7. Molkens, T., Rossi, B.: Modelling real fire by FDS and 2-zone model for structural post-fire assessment. In: Piloto, P.A.G., Rodrigues, J.P., Silva, V.P. (eds.) CILASCI 2019. LNCE, vol. 1, pp. 48-60. Springer, Cham (2020). https://doi.org/10.1007/ 978-3-030-36240-9_4

8. Rein, G., Bar-Ilan, A., Fernandez-Pello, C., Alvares, N.: A comparison of three fire models in the simulation of accidental fires. J. Fire Protect. Eng. 17 (2004)

9. Sacadura, J.: Radiative heat transfer in fire safety science, radiation IV proc. J. Quant. Spectrosc. Radiat. Transf. 93, 5-24 (2005). https://doi.org/10.1016/j.jqsrt. 2004.08.011

10. Walton, W.D., Carpenter, D.J., Wood, C.B.: Zone computer fire models for enclosures. In: Hurley, M.J., et al. (eds.) SFPE Handbook of Fire Protection Engineering, pp. 1024-1033. Springer, New York (2016). https://doi.org/10.1007/978-14939-2565-0_31

11. Wang, X., Fleischmann, C., Spearpoint, M.: Assessing the influence of fuel geometrical shape on fire dynamics simulator (FDS) predictions for a large-scale heavy goods vehicle tunnel fire experiment. Case Stud. Fire Saf. 5, 34-41 (2016). https:// doi.org/10.1016/j.csfs.2016.04.001

12. Yuan, W., Tan, K.H.: Cellular automata model for simulation of effect of guiders and visibility range. Curr. Appl. Phys. 9(5), 1014-1023 (2009). https://doi. org/10.1016/j.cap.2008.10.007. http://www.sciencedirect.com/science/article/pii/ S1567173908002733

13. Zheng, Y., Jia, B., Li, X.G., Zhu, N.: Evacuation dynamics with fire spreading based on cellular automaton. Phys. A 390(18), 3147-3156 (2011). https://doi.org/ 10.1016/j.physa.2011.04.011

14. Zheng, Z., Huang, W., Li, S., Zeng, Y.: Forest fire spread simulating model using cellular automaton with extreme learning machine. Ecol. Model. 348, 33-43 (2017). https://doi.org/10.1016/j.ecolmodel.2016.12.022 